\title{
A document level neural model integrated domain knowledge for chemical-induced disease relations
}

\author{
Wei Zheng ${ }^{1,2}$, Hongfei Lin ${ }^{1 *}$, Xiaoxia Liu ${ }^{1}$ and Bo Xu ${ }^{1 *}$
}

\begin{abstract}
Background: The effective combination of texts and knowledge may improve performances of natural language processing tasks. For the recognition of chemical-induced disease (CID) relations which may span sentence boundaries in an article, although existing CID systems explored the utilization for knowledge bases, the effects of different knowledge on the identification of a special CID haven't been distinguished by these systems. Moreover, systems based on neural network only constructed sentence or mention level models.

Results: In this work, we proposed an effective document level neural model integrated domain knowledge to extract CID relations from biomedical articles. Basic semantic information of an article with respect to a special CID candidate pair was learned from the document level sub-network module. Furthermore, knowledge attention depending on the representation of the article was proposed to distinguish the influences of different knowledge on the special CID pair and then the final representation of knowledge was formed by aggregating weighed knowledge. Finally, the integrated representations of texts and knowledge were passed to a softmax classifier to perform the CID recognition. Experimental results on the chemical-disease relation corpus proposed by BioCreative $\checkmark$ show that our proposed system integrated knowledge achieves a good overall performance compared with other state-of-the-art systems.
\end{abstract}

Conclusions: Experimental analyses demonstrate that the introduced attention mechanism on domain knowledge plays a significant role in distinguishing influences of different knowledge on the judgment for a special CID relation.

Keywords: Chemical-induced diseases, Document level, Knowledge, Attention mechanism, Neural network, Text mining

\section{Background}

Identifying chemical-disease relations (CDRs) are significantly crucial to improve some researches and applications in the biomedical and healthcare domains $[1,2]$. For example, it can contribute to biocuration of some bioinformatics databases such as Comparative Toxicogenomics Database $^{1}$ (CTD) [3, 4]. However, manual annotation of CDRs from literature is not only expensive but also difficult to catch up with the rapid literature growth $[4,5]$.

There has been currently an increased interest in exploiting computational approaches such as text-mining

\footnotetext{
*Correspondence: hflin@dlut.edu.cn; xubo@mail.dlut.edu.cn

${ }^{1}$ College of Computer Science and Technology, Dalian University of

Technology, Dalian, China

Full list of author information is available at the end of the article
}

techniques to automatically detect relations between biomedical entities. Therefore, the BioCreative V challenge included a task on automatical extraction of CDRs from curated Medline articles (only abstracts and titles). This challenge facilitates the identification of CDRs and promotes the development of text-mining techniques. In this task, all articles were manually annotated with chemical and disease mentions, their concept identifiers-MeSH ID (the identifier in Medical Subject Headings), and true chemical-induced disease (CID) relations within the scope of an article [6]. In the CDR corpus, nearly $1 / 3$ of all relations are described as inter-sentential CID relations [5]. Arguments of inter-sentential CID relations may cross sentence boundaries and never co-occur in the same sentence. This task remains difficult and challenging mainly 
because it requires recognizing inter- and intra-sentential causal relationships between chemical and disease concept identifiers (entities) rather than their special mentions (mention level) in an article.

The CID task is usually regarded as a binary classification problem. The current state-of-the-art systems [7-18] mainly use three types of methods: the traditional machine learning (ML) method, the rule-based method and the deep learning (DL) method. On the whole, those systems with a combination of knowledge bases (KB) and textual information outperform ones with textual information alone in performance. The importance of background knowledge in natural language understanding has been recognized [19-24]. Leveraging external knowledge to improve performances of natural language processing (NLP) applications attracts more and more researchers. In this work, what we are interested in is how to integrate knowledge bases with texts together to effectively learn the semantic representations of an article and improve performances of a DL-based CID system.

With the recent advances in deep learning technologies, the neural-network (NN) based systems in many NLP tasks, such as question answer, relation extraction and entity recognition, have obtained good performances due to the adaptively automatically learning capability for text representations. However, few systems exploit $\mathrm{NN}$ approaches to perform the CID task. Only the CNN-based mention level system [17] used knowledge from CTD and improved their F-score by $13.2 \%$. In addition, only two systems [12,13] without $\mathrm{KB}$ applied convolution neural network $(\mathrm{CNN})$ and recurrent neural network (RNN) to extract sentence level CID relations, respectively.

Most of systems [7-9, 11, 18] exploit traditional ML-based approaches such as support vector machine (SVM). Take the top-ranked system [9] during the BioCreative $\mathrm{V}$ evaluation for an example, its F-score changed from 50.73 to $67.16 \%$ after exploiting features from four types of knowledge bases including MeSH, Side Effect Resource (SIDER), MEDication Indicaton Resource (MEDI) and CTD. Similarly, Pons et al. [8] made use of a graph database which contains entities and relations from (curated) structured databases (UniProt, CTD and UMLS) and from scientific abstracts. In addition to using knowledge features derived from some databases, these systems also extracted the sentence level and document level features. The sentence level features derived from a sentence usually include various lexical and syntactic features. The document level features related to chemicals and diseases often consist of information of relevant sentences, statistical features, high-frequent entities and trigger words. Besides the SVM-based systems, the rule-based system [10] achieved competitive performances. This system built a disease dictionary derived from $\mathrm{MeSH}$, the disease ontology and Wikipedia. Furthermore, the system [7] combining the advantages of rule- and ML-based approaches not only used features from CTD but also augmented their training data from existing curated data of the CTD-pfizer collaboration. However, since these systems depend on specialized designs of domain experts for features or rules, it is difficult to generalize them to other relation extraction tasks.

In summary, one of the reasons for good performances of the above all systems with $\mathrm{KB}$ in the CDR task may be due to the direct or indirect exploitation of CTD. In these systems, chemical-disease relationships from CTD serve as features during machine learning. CTD provides four types of manually curated chemical-disease relations which often are called as knowledge in the subsequent sections.

However, whether SVM-based systems or NN-based systems, they all didn't distinguish the effects of different knowledge on the CID judgement. SVM-based systems $[7-9,11]$ took advantages of knowledge either as features of equal importance or as Boolean features, while the NN-based system [17] concatenated one-hot representations of knowledge as a feature of the model indiscriminately. Because these relations in CTD are in nature different from each other, it is impossible for them to make the same contribution to assisting a classifier to recognize a CID relation. Therefore, a system employing chemical-disease relations from CTD should make a distinction between the influences of different knowledge on identifying a special CID according to the semantic meaning of an article. Accordingly, its model should learn the representations of texts and knowledge in a way of interdependence rather than in isolation.

In this work, because of the above mentioned two reasons, we explored the issue of how to distinguish the influences of different knowledge on the judgment of a special CID relation when knowledge is used as features to incorporate into a NN-based model. Currently, attention-based models have shown great success in many NLP tasks such as question answering [24, 25], machine translation [26, 27] and relation extraction [2830]. In the context of relation classification, by learning a scoring function to weigh concerned feature representations, attention mechanism allows a model to pay more attention to the most influential representations for a relationship category. Thus, the different knowledge from CTD may be weighed by a scoring function depending on the semantic representation of an article. Consequently, mutual influences between texts and knowledge can be revealed because of the exploiting of attention mechanism.

Overall, the contributions of this work are as follows. (1) We proposed an effective document level model incorporated domain knowledge to detect CID relations from biomedical articles. (2) A knowledge attention depending on the learned semantic representation of an 
article was proposed to distinguish the influences of different relations from CTD on identifying a special CID. On this basis, the final representation of knowledge was formed by aggregating weighed relations. (3) The high level representations of an article and knowledge were further weighted to evaluate their importance to final classifying results.

The experimental results on the CDR corpus demonstrate that the proposed system integrated KB are highly competitive compared with other state-of-the-art CID systems in spite of the use of less features. Moreover, experimental analyses indicate that the introduced attention mechanism on knowledge may not only distinguish the influences of different knowledge on recognizing special CID relations but also improve the performances of the proposed system.

\section{Methods}

In the section, text processing adopted in the proposed system is first introduced. Next, an overview of the network architecture is shown. Then, the hierarchical document level sub-network module and knowledge with attention mechanism are described in detailed, respectively.

\section{Text processing}

Appropriate text processing in NLP tasks may generally improve performances of a system to some extent. In the proposed model, the following processing operations were applied to articles of all datasets. Numbers (integers and decimals) without letters were transformed into a special token. The MeSH ID of a disease (or a chemical) substituted for the corresponding mentions. In addition, since each candidate entity often occurs multiple mentions in an article, it is crucial for a document level model to distinguish between candidate entities and other tokens of an articles to pick up the contexts more specifically. Therefore, special marks were employed to indicate the mentions of different candidate entities. For example, in the replaced sentence "The precipitating cause of ds_d012640 was believed to be a $d s \_s t a r t$ ds_d062787 ds_end of ch_start ch_d014148 ch_end", substrings "ch" and " $d s$ " are used to distinguish between the chemical and the disease; substrings "d014148" and "d062787" are MeSH IDs of the replaced chemical and disease, respectively; substrings "_start" and "_end" represent the beginning and end of each candidate entity, respectively. Finally, each article was divided into sentences and each sentence was parsed by our improved Standford CoreNLP Tool [31] to get the PoS (Part of Speech) tag of each word.

\section{Network architecture}

Both knowledge representation derived from CTD and the semantic representation learned from an article will play an important role for judging the relationship of a special candidate pair. Therefore, a model should have the ability to discern which knowledge is more influential to the considered pair when it learns the semantic meaning effectively and automatically from the original text segments. Moreover, the two types of representations might have the different effects on the recognition of a chemical-disease relation. On these grounds, Fig. 1 gives an overview of the network architecture. Each article is inputted to the proposed model by sentence sequences. The main layers of the proposed model are as follows: (1) the document level hierarchical sub-network to learn the basic semantic meaning of a candidate pair only from the original text segments of an article, which is implemented by learning the semantic representation of each sentence, relations among sentences and the theme of an article; (2) the embedding layer to look up
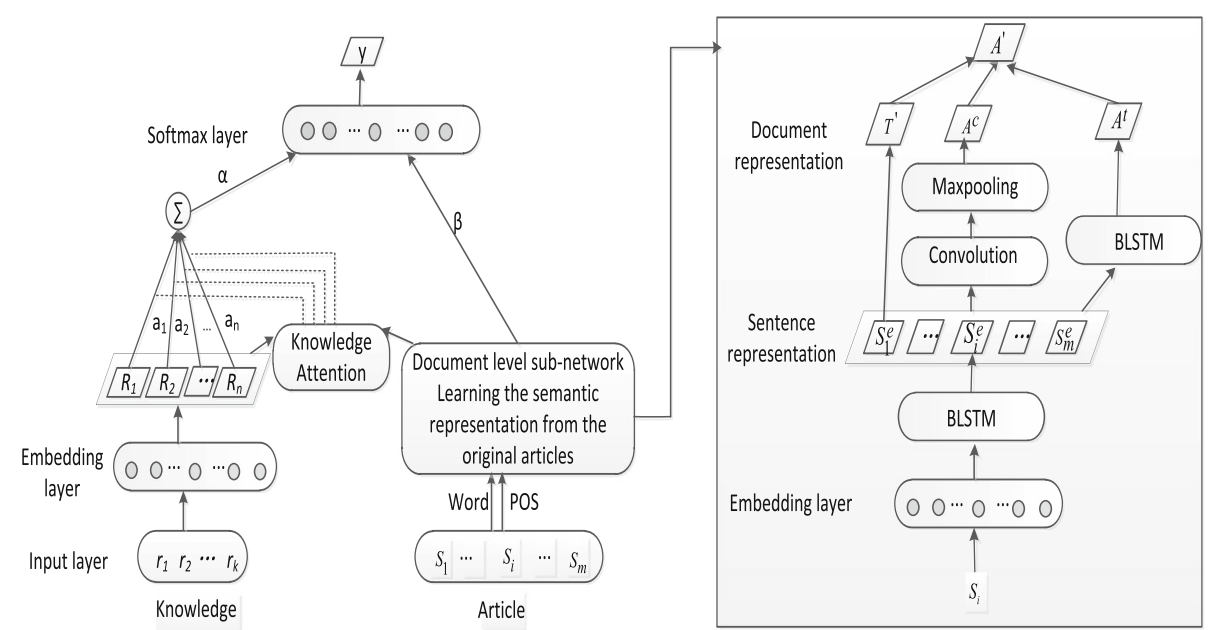

Fig. 1 The overall architecture of the proposed model 
the knowledge embedding vocabulary to encode relations of CTD into vectors; (3) knowledge attention to act the semantic representation of an article on the different knowledge candidates to highlight the most influential relations for the candidate pair; (4) weighted relations to be aggregated to serve as the final knowledge representation for a given pair; (5) representations of texts and knowledge to be weighted to reflect their different effect on final classifying results; (6) the softmax layer to conduct relation classification according to the above combined semantic meanings.

\section{Input representations}

Given an article with $\mathrm{n}_{1}$ sentences $D=\left\{S_{1}, S_{2}, \ldots, S_{i}, \ldots\right.$, $\left.S_{\mathrm{n}_{1}}\right\}$, each sentence $S_{\mathrm{i}}=\left\{w_{1}, w_{2}, \ldots, w_{\mathrm{j}}, \ldots, w_{\mathrm{n}_{2}}\right\}$ has a maximum of $\mathrm{n}_{2}$ words. Since word embedding [32] maps words to low-dimensional real space where semantic meanings of words can be represented by vectors, the embedding layer of the proposed model will look up the embedding vocabulary to perform this transformation process according to the corresponding index of each input token. Here, each embedding vocabulary can be initialized either by a random process or by some pre-trained word embedding vectors.

(1) Word and PoS: In the proposed model, the semantic meaning of each word $w_{\mathrm{j}}$ is represented by concatenating the corresponding $l_{1}$-dimension word embedding vector $\boldsymbol{w}_{\mathrm{j}}^{\mathrm{e}}$ and $l_{2}$-dimension PoS (part of speech) embedding vector $\boldsymbol{p}_{\mathrm{j}}^{\mathrm{e}}$. The PoS feature of a word is valuable for relation classification tasks [28]. After the word $w_{j}$ is passed through the embedding layer, it is denoted as a new vector $W_{\mathrm{j}}=\left[\boldsymbol{w}_{\mathrm{j}}^{\mathrm{e}} ; \boldsymbol{p}_{\mathrm{j}}^{\mathrm{e}}\right] \boldsymbol{W}_{\mathrm{j}} \in R^{l}\left(l=l_{1}+l_{2}\right)$ where the symbol ";" means the concatenation operation. Hence, the sentence $S_{\mathrm{i}}$ is represented as an array $S_{\mathrm{i}}^{\mathrm{e}}=\left[\boldsymbol{W}_{1}, \boldsymbol{W}_{2}, \ldots, \boldsymbol{W}_{\mathrm{j}}, \ldots, \boldsymbol{W}_{\mathrm{n}_{2}}\right]$.

(2) Knowledge: For a pair of chemical and disease, it has at most four types of relations in CTD, namely "marker/mechanism", "theapetic", "infered" and "null". Thus, knowledge about relations is denotes as $R=\left\{r_{1}, r_{2}, \ldots, r_{\mathrm{k}}, \ldots, r_{\mathrm{n}_{3}}\right\}\left(\mathrm{n}_{3}=4\right)$. If the number of relations extracted from CTD is less than $n_{3}$, the fixed-length representation will be obtained through padding with the relation "null". By looking up the knowledge embedding vocabulary to obtain the $m$ dimension embedding vector $\boldsymbol{r}_{\mathrm{k}}^{\mathrm{e}}$ of each relation $r_{\mathrm{k}}$, knowledge $R$ is denoted as an array $\boldsymbol{R}^{\mathrm{e}}=\left[\boldsymbol{r}_{1}^{\mathrm{e}}, \boldsymbol{r}_{2}^{\mathrm{e}}, \ldots\right.$, $\left.r_{\mathrm{k}}^{\mathrm{e}}, \ldots, r_{\mathrm{n}_{3}}^{e}\right]$.

\section{The document level sub-network}

As the above mentioned, the CDR corpus consists of two types of CID relations: intra- and inter-sentential relations. Candidate entities in inter-sentential CID relations may occur either among the adjacent sentences or among the nonadjacent sentences. A true CID relation is recognized according to the theme of an article, regardless of whether it is an intra-sentential relation or an inter-sentential relation. The document level hierarchical sub-network is applied to adapt to these characteristics of the CDR corpus.

(1) The semantic meaning of sentences and the theme of an article

Above all, the CDR corpus contains a great number of long sentences with the more complicated structure compared with corpora of the general domain. RNN [33], especially RNN with long short term memory (LSTM) units [34], has been demonstrated to suit many NLP tasks. LSTM is superior in capturing unbounded contexts due to the introduction of the gating mechanism, especially when it is used to model variable length of long texts. However, the LSTM's hidden state $h_{\mathrm{t}}$ collects contexts only from the previous words (the past) and knows nothing about the subsequent texts (the future). Therefore, for the sentence $S_{i}$ of an article, the proposed model makes use of a bidirectional LSTM (BLSTM) which is composed of forward and backward LSTM. BLSTM can capture past and future contextual informaiton of the current word. Hidden states $\left(\overleftarrow{\boldsymbol{h}_{\mathrm{n}_{2}}}\right.$ and $\overrightarrow{\boldsymbol{h}_{\mathrm{n}_{2}}}$ ) of the two LSTMs at the last time step $\mathrm{n}_{2}$ are concatenated to form a new vector $S_{\mathrm{i}}^{\prime}=\left[\overrightarrow{\boldsymbol{h}_{2}} ; \overleftarrow{\boldsymbol{h}_{\mathrm{n}_{2}}}\right]$ which is regarded as the represention of the sentence $S_{i}$. Thus, all sentences of the document $D$ are denoted as an array $D^{\mathrm{e}}=\left[S_{1}^{\prime}, S_{2}^{\prime}, \ldots, S_{\mathrm{i}}^{\prime}, \ldots, S_{\mathrm{n}_{1}}^{\prime}\right]$.

In addition, the theme of an article is expressed by the semantic meaning of the title of the article which is usually a sentence. Likewise, utilizing the BLSTM network learns the representation $\boldsymbol{T}$ of the theme of an article.

\section{(2) The semantic meaning of an article for a given pair}

Furthermore, two types of sub-networks are constructed on the representation $D^{\mathrm{e}}$ of all sentences to capture the document level semantic meaning of a given candidate pair within the scope of an article. The one is the BLSTM network on all sentences, which captures the temporal-based dependency $\boldsymbol{A}^{\mathrm{t}}$ among nonadjacent sentences. The other one is the CNN network on all sentences, which extracts local contexts among adjacent sentences. $\mathrm{CNN}$ is prone to capturing the local features to generate an informative latent semantic representions of text segments such as the sentence and the paragraph. In the proposed model, a convolution layer involves $f$ filters which are applied to a window of $w$ sentences to 
obtain the representation $\boldsymbol{L C}$ of local dependencies. Subsequently, a max pooling operation on the representation $L C$ collects the global significant contexts to produce the document level representation $A^{\mathrm{C}}$ of the candidate pair. Similar to Collobert et al. [35], the definition of the equations is as follows:

$$
\begin{aligned}
\boldsymbol{L} \boldsymbol{C} & =\operatorname{ReLU}\left(\boldsymbol{W}_{\mathrm{c}} \boldsymbol{D}^{\mathrm{e}}+\boldsymbol{b}_{\mathrm{c}}\right) \\
\boldsymbol{A}^{\mathrm{c}} & =\max \boldsymbol{L C}(\cdot, i) \quad 0 \leq i<f
\end{aligned}
$$

Where $W_{\mathrm{c}}$ is the learned matrix, $\boldsymbol{b}_{\mathrm{c}}$ is a bias vector, $\boldsymbol{L C}(\cdot, i)$ denotes the $i$-th column of the matrix $\boldsymbol{L C}$, and $R e L U$ means the rectified linear activation function. So far, for the two types of inter-sentential CIDs, the sub-network has the ability to capture the relevant contexts by exploiting the different advantages of $\mathrm{CNN}$ and LSTM in pattern learning.

Finally, the three document level vectors are concatenated to represent the semantic meaning of the given pair in an article, which is denoted as $A^{\prime}=\left[A^{\mathrm{t}} ; A^{\mathrm{c}} ; \boldsymbol{T}^{\prime}\right]$.

\section{Knowledge with attention mechanism}

Attention mechanism has been successfully applied to some NLP tasks. The CDR task requires classifying the relation between a pair of candidate chemical and disease according to the discussed topic of an article. It is obvious that not all relations of CTD have equal contributions to helping to determine the relationship type of the candidate pair. Therefore, it is necessary for each relation from CTD to learn a weight to reflect its level of effect on the final classification. Since the relation type of a given pair mainly relies on the semantic meaning of an article, acting the semantic meaning of the article on each relation from CTD may highlight which relation from CTD is the most influential for the considered pair. For this purpose, the proposed model applies attention mechanism to original knowledge vectors for weighing each relation in CTD. We exploit the item $\alpha_{\mathrm{k}}$ of a row vector $\alpha$ to quantify the relevance degree of each relation $r_{\mathrm{k}}$ from CTD with respect to the semantic meaning $\boldsymbol{A}^{\prime}$ of an article, the related equations are defined as follows:

$$
\begin{aligned}
& \alpha_{\mathrm{k}}=\frac{\exp \left(s\left(\boldsymbol{A}^{\prime}, \boldsymbol{r}_{\mathrm{k}}^{\mathrm{e}}\right)\right)}{\sum_{\mathrm{k}^{\prime}=1}^{\mathrm{n}_{3}} \exp \left(s\left(\boldsymbol{A}^{\prime}, \boldsymbol{r}_{\mathrm{k}^{\prime}}^{\mathrm{e}}\right)\right)} \\
& s\left(\boldsymbol{A}^{\prime}, \boldsymbol{r}_{\mathrm{k}}^{\mathrm{e}}\right)=\frac{\boldsymbol{A}^{\prime} \boldsymbol{W} \boldsymbol{r}_{\mathrm{k}}^{\mathrm{e}}}{m} \\
& \boldsymbol{r}_{\mathrm{k}}^{\prime}=\boldsymbol{r}_{\mathrm{k}}^{\mathrm{e} *} \alpha_{\mathrm{k}}
\end{aligned}
$$

Here, $s\left(A^{\prime}, r_{\mathrm{k}}^{\mathrm{e}}\right)$ is the score function, $\boldsymbol{W}$ is the learned weight matrix and $m$ is the dimensionality of a knowledge vector. The dot-product operation is used to perform the calculation in Eq. (4). The new representation $\boldsymbol{r}_{\mathrm{k}}^{\prime}$ of each relation from CTD is calculated by the element-wise multiplication between its original embedding vector $r_{\mathrm{k}}^{\mathrm{e}}$ and the corresponding weight $\alpha_{\mathrm{k}}$. Then, the final representation of knowledge is derived from the aggregating effect ATT_KB_Sum of all relations from CTD:

$$
\boldsymbol{K}^{\prime}=\sum \boldsymbol{r}_{\mathrm{k}}^{\prime}
$$

For the sake of comparison, we still provide other two types of knowledge representations including ATT_KB_Max and ATT_KB_Con:

$$
\begin{aligned}
& \boldsymbol{K}^{\prime}=\boldsymbol{R}^{\mathrm{e}}\left(\operatorname{argmax}\left(\alpha_{\mathrm{k}}\right), \cdot\right) \\
& \boldsymbol{K}^{\prime}=\underset{\mathrm{k}}{\operatorname{con}\left(\boldsymbol{r}_{\mathrm{k}}^{\prime}\right)}
\end{aligned}
$$

Where $\boldsymbol{R}^{\mathrm{e}}\left(\operatorname{argmax}\left(\alpha_{\mathrm{k}}\right),.\right)$ denotes a row of the matrix $\boldsymbol{R}^{\mathrm{e}}$ which corresponds to the relation with the maximum weight $\alpha_{\mathrm{k}}$, and the symbol "con" denotes the concatenating operation acting on all knowledge vectors $\boldsymbol{r}_{\mathrm{k}}^{\prime}$.

\section{Training and classification}

The softmax layer performs relation classification for a pair of candidate chemical and disease. After weighted representations of texts and knowledge are concatenated, the new vectors $D_{s}$ will be passed to the softmax layer. And then, the probability distribution over each category will be output.

$$
\begin{aligned}
& \boldsymbol{D}_{\mathrm{s}}=\left[\beta_{1} \boldsymbol{A}^{\prime} ; \beta_{2} \boldsymbol{K}^{\prime}\right] \\
& p(y=t \mid D)=\operatorname{soft} \max \left(\boldsymbol{D}_{\mathrm{s}} \boldsymbol{W}_{\mathrm{s}}+\boldsymbol{b}_{\mathrm{s}}\right) \\
& \hat{y}=\arg \max _{y \in C}(p(y=t \mid D))
\end{aligned}
$$

Where $\beta_{1}$ and $\beta_{2}$ denotes weights, $W_{\mathrm{s}}$ is a weigh matrix, $\boldsymbol{b}_{\mathrm{s}}$ is a bias vector, $t$ is the label of a category, and $\hat{y}$ denotes the predicted label of a candidate pair. The training objective is cross-entropy cost function and RMSprop (Resilient Mean Square Propagation) [36] is used to update parameters with respect to the cost function.

\section{Post processing}

The CID task is concerned with the relations between the most specific diseases and chemicals in an article. For example, the kidney disease (general/ hypernymy) vs. chronic kidney failure (special/ hyponymy), if a chemical and chronic kidney failure hold a CID relation, the chemical and the kidney disease may not been annotated as a CID relation even if they have a semantic induced relation. Only relying on machine learning automatically may 
result in wrong judgements. Therefore, similar to our previous work [37], if an article includes specific diseases than a disease $d_{i}$ which does not appear in the title, extracted chemical-disease pairs with the disease $d_{i}$ are seen as negative instances. The hypernymy/hyponymy relations among diseases may be calculated by MeSH Tree Number.

\section{Results and discussion}

\section{Dataset and evaluation settings}

The CDR corpus [6] consists of a total of 1500 Medline articles: 500 each for the training, development and test set. For each given article of the CDR corpus, we first constructed relation instances because each article only annotates real CID relations. Candidate pairs <chemical $\mathrm{MeSH}$ ID, disease MeSH ID > were generated by matching chemical and disease entities co-occurring in an article. Moreover, entities of the inter-sentential candidate pairs were limited to co-occurrance within $K$ consecutive sentences to avoid selecting unlikely candidates. Furthermore, if a candidate pair hasn't been annotated as a CID relation in a given article, it will be labeled as negative. Table 1 shows the statistics of the constructed candidate pairs.

Next, we combined the original training set with the development set to argument the training set due to the limited number of samples of the CDR corpus. Similar to the common training approach of samples in NN-based systems, the union set was randomly divided into 10 equal subsets, one of which was for the new development set and the others of which all were for the new training set. The test set is still original. The minimum sentence span $K$ strategy $(K=4$ based on our previous work) only was applied to the new development and the original test datasets because of the above mentioned same reason. In addition, some real CID relations filtered by this strategy were treated as false negative instances.

The performances of the proposed model were assessed by the standard evaluation measures: precision $(\mathrm{P})$, recall (R) and F-score (F). Furthermore, gold standard entities of the CDR corpus were employed to objectively evaluate each related model in this task because named entity recognition has the strong effect on the classifying performances. We

Table 1 The statistics of the CDR corpus

\begin{tabular}{lllll}
\hline Dataset & CID pairs & CD pairs & $\begin{array}{l}\text { Inter-sentential } \\
\text { CID pairs }\end{array}$ & $\begin{array}{l}\text { Intra-sentential } \\
\text { CID pairs }\end{array}$ \\
\hline Training & 1038 & 5432 & 283 & 755 \\
Development & 1012 & 5263 & 246 & 766 \\
Test & 1066 & 5405 & 303 & 763 \\
Total & 3116 & 16,100 & 832 & 2284
\end{tabular}

The column " $C D$ pairs" represents the total number of candidate instances used Keras library with theano backend to implement the proposed model.

\section{The pre-training corpora of embedding vectors}

With respect to the training corpus for domain knowledge, since most articles (1400) of the CDR corpus come from the related CTD-Pfizer dataset, we downloaded the package "CTD_chemicals_diseases.xml.gz" from the CTD database and extracted the corresponding chemical MeSH ID, the disease MeSH ID and their relationship for all chemical-disease pairs (2,048,652 pairs). The CTD database provides with manually curated interactions between chemical, gene and disease. After that, TransE $E^{3}$ implemented by Tsinghua University was used to train the extracted triples and generate the embedding vectors of entities and relations. TransE [38] is an effective approach when it deals with embedding a large scale knowledge graph composed of entities and relations into a continuous vector space. The proposed model only exploited relation vectors.

Articles of the bioconcepts package (bioconcepts2pubtator_offsets.gz, about 22 gigabytes) downloaded from PubTator $^{4}$ [39] were used as the training corpus of the word representation. The training corpus of the PoS representation comes from one fifth of texts randomly chosen from the above training corpus of the word representation. The word2vec tool ${ }^{5}$ [40] was employed to train the above two corpora and output word and PoS embedding vectors, respectively.

\section{Hyperparameters}

We tuned the hyperparameters on the new development set (the subset with the index 0) to optimize performances of the proposed model. Table 2 lists these parameters and their corresponding values used in the proposed model.

The proposed model was tested with different dimensions of word embedding. Figure 2 shows that the 100-dimension word embedding makes the system achieve the highest

Table 2 Hyperparameters

\begin{tabular}{ll}
\hline Parameter Name & Value \\
\hline I: Word emb.Size & 100 \\
I2: POS emb.Size & 10 \\
m: Knowledge emb.Size & 200 \\
The number $n_{1}$ of sentences in an article & 30 \\
The number $\mathrm{n}_{2}$ of words in a sentence & 120 \\
The window size $w$ for CNN & 5 \\
The number $f$ of filters for CNN & 300 \\
Mini-batch & 8 \\
The number of hidden units of two LSTMs & 220,440 \\
The learning rate $I r$ of RMSprop & 0.001 \\
The dropout rate & 0.5 \\
\hline
\end{tabular}




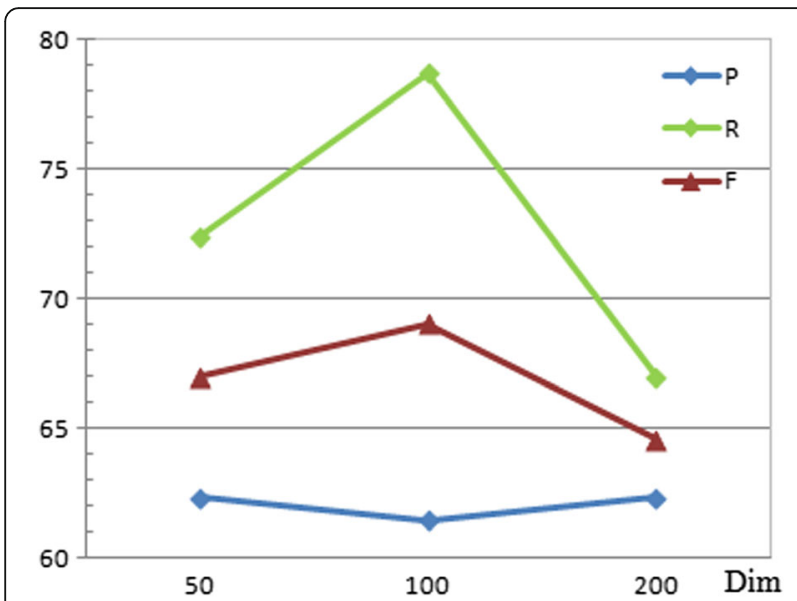

Fig. 2 Performance evaluation for the dimension of the word embedding on the test set of the CDR corpus

F-score. The dimension of PoS embedding was set as 10 as used by Zeng [41]. Based on the statistics of CDR texts, each article includes up to $30\left(\mathrm{n}_{1}\right)$ sentences and each sentence contains a maximum of $120\left(\mathrm{n}_{2}\right)$ words. In addition, the evaluation for the dimension of knowledge vectors is shown in Fig. 3. The proposed system obtains the best F-score when the dimension of knowledge vectors is 200. Furthermore, two initialization methods of knowledge embedding vectors including random and TransE were compared to evaluate their impact on performances of the proposed system. Table 3 shows that using knowledge vectors trained by TransE makes the system obtain the higher precision and F-score than that by random. The reason might be due to the fact that the TransE method exploiting a large scale knowledge graph brings knowledge embedding vectors more targeted semantic meanings than the random method.

The numbers (220 and 440) of hidden units of two LSTM layers are equal to the size of their corresponding input dimensions in order to simplify the research process. Considering that two sentences before

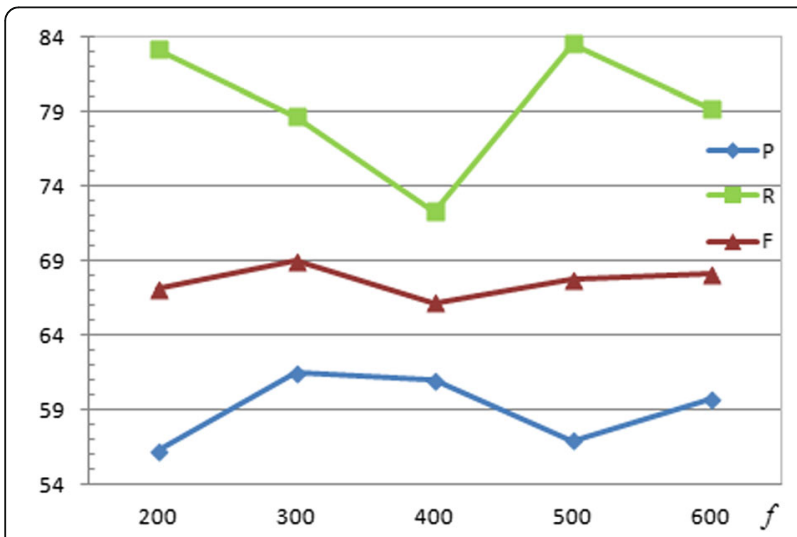

Fig. 3 Performance evaluation for the number $f$ of filters on the test set of the CDR corpus
Table 3 Performance evaluation for different initialization methods of the knowledge embedding on the test set of the CDR corpus

\begin{tabular}{llll}
\hline Methods & $P(\%)$ & $R(\%)$ & $F(\%)$ \\
\hline Random & 57.1 & 81.0 & 67.0 \\
TransE & 61.5 & 78.7 & 69.0 \\
\hline
\end{tabular}

The post processing step wasn't applied to the experimental results in this table

and after the current sentence may generally embody the semantic meaning of the inter-sentential candidate pair, we empirically set the window size $w=5$. As shown in Fig. 4, the proposed system achieves a good F-score when the number $f$ of filters in $\mathrm{CNN}$ is 300. The mini-batch was set as 8 . The learning rate $l r$ of RMSprop was set as 0.001 as suggested by Tieleman et al. [36]. The dropout strategy was applied on the LSTM and softmax layers to prevent the over-fitting problem, respectively. The dropout rate was assigned to 0.5 as suggested by Hinton et al. [42].

\section{Effects of input representations and the architecture}

In NLP tasks, input features and post processing may partly influence performances of a system. Table 4 lists their effects on performances of the proposed system.

Table 4 shows that the proposed system achieves an F-score of $57.7 \%$ when it takes only the word embedding as input. When knowledge from CTD is incorporated into the proposed model, the F-score of the system increases by $8.6 \%$, which demonstrates that the model which integrates domain knowledge with the semantic meaning of an article may effectively promote performances of the proposed system. The effect of domain knowledge will further be analysed in the following section. Furthermore, with the introduction of the PoS feature, the precision, the recall and the F-score all are improved, which indicates that PoS tags contain a certain amount of effective information for identifying relations. Finally, post processing applied appropriately in

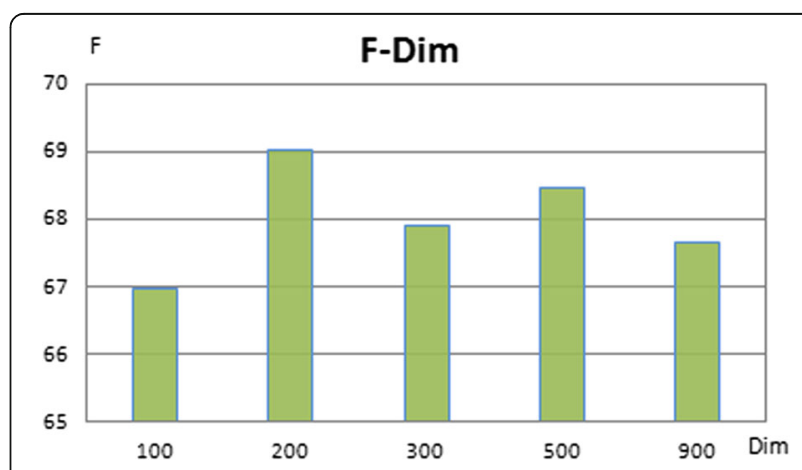

Fig. 4 Performance evaluation for the dimension of the knowledge embedding on the test set of the CDR corpus 
Table 4 Performance changes with different input representations and post processing on the test set of the CDR corpus

\begin{tabular}{llll}
\hline Feature & $P(\%)$ & $R(\%)$ & $F(\%)$ \\
\hline (1): word & 52.0 & 64.9 & 57.7 \\
(2): (1)+ CTD & 59.5 & 74.8 & 66.3 \\
(3): (2) + POS & 61.5 & 78.7 & 69.0 \\
(4): (3) + PP & 64.4 & 76.7 & 70.0 \\
\hline
\end{tabular}

the proposed system improves the precision and F-score to some extent.

Besides, Table 5 lists the performance changes with different components of the document level sub-network (see the right section of Fig. 1) on the test set of the CDR corpus when knowledge isn't incorporated into the proposed model.

\section{Effects of knowledge with attention mechanism}

(1) The final representation of knowledge

Knowledge obviously contributes to the performance improvement in many NLP tasks. As mentioned above, there are four types of relations in CTD. In the proposed model, knowledge associates with the semantic meaning of an article together to perform the CID classification. Therefore, it is crucial to make the final representation of knowledge play its role more effectively. Table 6 lists different final representations of knowledge and related performances on the test set of the CDR corpus. In this table, the prefix string "ATT_KB_" denotes a model employing the proposed attention mechanism.

On the whole, except for "ATT_KB_Max", models exploiting knowledge with attention mechanism obtain the better recall and F-score than the corresponding models without attention mechanism. Compared with the approaches "Sum" and "Con" without attention mechanism, "ATT_KB_Sum" and "ATT_KB_Con" make the F-score increase by 1.2 and $0.6 \%$, respectively. Among all approaches, "ATT_KB_Sum" achieves the best F-score. For the approach "ATT_KB_Con", the expanded dimension of the knowledge representation

Table 5 Performance changes with different components of the document level sub-network on the test set of the CDR corpus when knowledge isn't incorporated

\begin{tabular}{llll}
\hline Architecture & $P(\%)$ & $R(\%)$ & $F(\%)$ \\
\hline (1): Istm+Istm & 48.0 & 62.8 & 54.4 \\
(2): Istm+cnn & 54.8 & 59.2 & 56.9 \\
(3): Istm+cnnlstm & 56.5 & 61.3 & 58.8 \\
(4): Istm+cnnlstm+topic & 54.3 & 65.9 & 59.5 \\
\hline
\end{tabular}

The post processing step wasn't applied to the experimental results in this table
Table 6 Performance changes with the different final representations of knowledge on the test set of the CDR corpus

\begin{tabular}{llll}
\hline Methods & $P(\%)$ & $R(\%)$ & $F(\%)$ \\
\hline (1): Without KB & 54.3 & 65.9 & 59.5 \\
(2): Con & 61.5 & 74.9 & 67.5 \\
(3): Sum & 65.3 & 70.4 & 67.8 \\
(4):ATT_KB_Con & 59.6 & 79.5 & 68.1 \\
(5): ATT_KB_Max & 60.6 & 69.9 & 64.9 \\
(6): ATT_KB_Sum & 61.5 & 78.7 & $\mathbf{6 9 . 0}$
\end{tabular}

The post processing step wasn't applied to the experimental results in this table. The highest F-score is highlighted in bold

derived from the concatenating operation is closer to the dimension of the semantic meaning of the article. Consequently, the redundant noise information brought by the knowledge presentation without any processing slightly weakens the learning capacity of the model. On the contrary, "ATT_KB_Sum" not only retains the proper dimension of the knowledge presentation but also highlights and fuses the most relevant knowledge representations related to a special article. This reason might also explain why "ATT_KB_Max" doesn't achieve a relatively good performance. "ATT_KB_Max" only picks up the relation with the maximum weight as the final knowledge representation. On this basis, if an ineffective or wrong knowledge is learned, the model might partly be misled to make the wrong judgment for the relation type.

(2) Learned attention values

In addition, we manually examined the weights (attention values) of four relation types for all instances of the test set. The CID relations mainly refer to two types of relations between a chemical and a disease in the CTD task: putative mechanistic relationships and biomarker relationships. Therefore, the relation type "marker/mechanism" in CTD shows more obvious weight change than the other relation types because of its strong informativity. This result indicates that the type "marker/mechanism" makes a significant contribution to recognizing CID relations. Among the other three relation types, the relation types "infered" and "null" have the nearly weights. Accordingly, they play the minor effect on relation extraction of CID. The weight change of the type "theapetic" is at the intermediate level among all relation types.

Figure 5 shows the weight of each relation learned by the proposed model with the approach "ATT_KB_Sum" for a true CID candidate (D007213 and D007022 from Doc ID 439781 in the test set) and not a true CID candidate (D009538 and D003866 from Doc ID 24114426 in the test set). The two candidate pairs contain all four types of relations in CTD. It can be seen from Fig. 5 that the relation type "marker/mechanism" has the relatively 


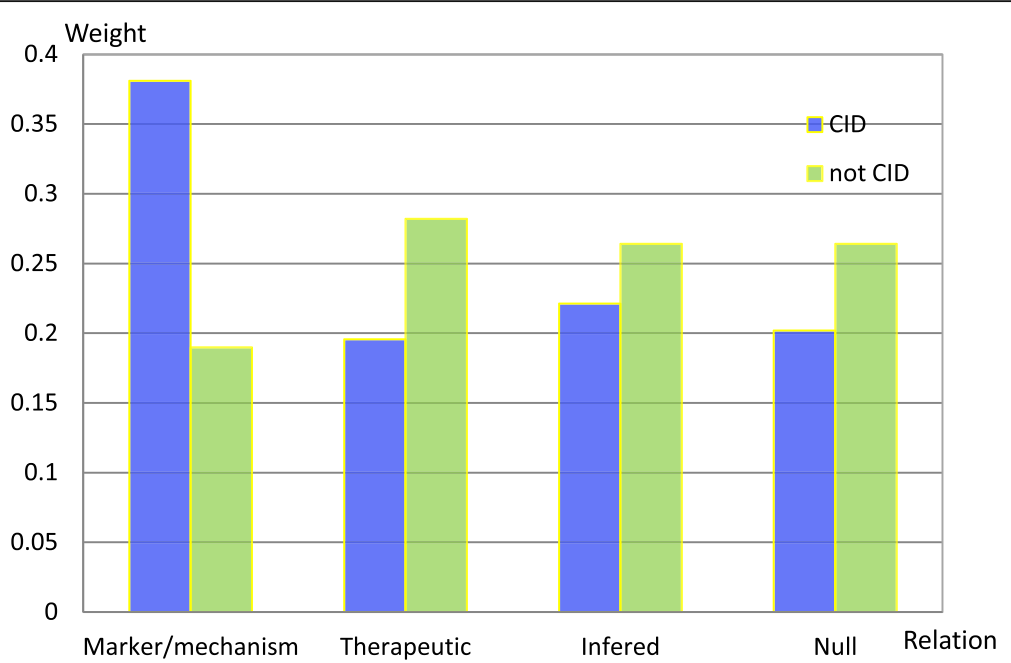

Fig. 5 Attention value learned by the model with the approach "ATT_KB_Sum" for chemical and disease pairs

higher weight than other relation types for the true CID, while the weight of the relation type "therapeutic" is relatively higher for the not true CID. These results seem to agree with the semantic meanings of the corresponding articles. For the article containing the aboved true CID, indomethacin induced hypotension in sodium and volume depleted rats. In contrast, the article containing the above not a true CID candidate only mentions the experiments related to nicotine and depression. Hence, with respect to the recognition of the candidate relation, it might be inferred that the proposed model can learn more beneficial representations from domain knowledge bases to some extent by introducing attention mechanism targeting the document level semantic meaning of an article.

Furthermore, we assigned different weights $\left(\beta_{1}\right.$ and $\left.\beta_{2}\right)$ to semantic representations of an article and knowledge. Experimental results indicate that the learned weights didn't improve system performances. Therefore, these two values were assigned as 1 for each candidate pair.

\section{Performance comparisons with other systems}

To evaluate our approach, we compared the proposed model mainly with the relevant models with gold standard entity annotations on the CDR corpus. Table 7 lists performances and relevant descriptions of these systems. In particular, we used each of the ten subsets as a development set and finished CID classifications on the original test dataset in turn. The average performances of ten experimental results were shown in Table 7. The standard deviation $\sigma \mathrm{F}$ of $\mathrm{F}$-scores is $0.67 \%$ and $0.49 \%$ before and after post processing, respectively.

These systems are divided into two groups: with $\mathrm{KB}$ and without KB. Obviously, most systems with $\mathrm{KB}$ have higher F-score than those without KB except two systems. This result further demonstrates that the effective combination of textual information and domain knowledge would improve performances of many CID systems.

For two types of CID systems including the tradition al-ML-based systems and the NN-based systems, the NN-based systems can automatically learn semantic representations of text segments and domain knowledge, while the traditional-ML-based systems commonly rely on carefully handcrafted features, elaborately designed kernels and statistical features.

\section{(1) Comparison with NN-based systems}

Among NN-based systems, the proposed system "ATT_KB_sum" achieves the best precision and F-score. Verga et al. [16] encoded full paper abstracts using an efficient self-attention encoder and formed pairwise predictions between all mentions with a bi-affine operation. Moreover, they improved the system performances by adding extra PubMed abstracts annotated in the CTD-pfizer dataset to their training set as Peng et al. [7] did. The chemical-disease relations from CTD were not directly applied to their system. Conversely, $\mathrm{Li}$ et al. [17] and our system incorporated knowledge from CTD with the semantic meaning of texts. However, Li et al. integrated knowledge only in a simple way, despite that their system achieved better performances. They used a hidden layer to covert one-hot representations of all knowledge into dense real value vectors which will be further concatenated with the semantic meaning of texts related to the nearest chemical and disease pair. They didn't distinguish the influences of different relation types from CTD on a given chemical-disease candidate in different articles. On the contrary, attention mechanism in our system 
Table 7 Performance comparisons with relevant systems using gold standard entity annotations on the test dataset of the CDR corpus

\begin{tabular}{|c|c|c|c|c|c|c|}
\hline Methods & System & Methods & Text and concept level & $P(\%)$ & $R(\%)$ & $F(\%)$ \\
\hline \multirow[t]{6}{*}{ NN with $K B$} & \multirow[t]{2}{*}{ ATT_KB_sum } & LSTM+CNN + CTD & \multirow[t]{2}{*}{ Doc_E } & 60.7 & 78.7 & 68.5 \\
\hline & & $\mathrm{LSTM}+\mathrm{CNN}+\mathrm{CTD}+\mathrm{pp}$ & & 63.6 & 76.8 & 69.6 \\
\hline & \multirow[t]{2}{*}{ Li et al. [17] } & CNN & \multirow[t]{2}{*}{ Doc_M } & 57.8 & 54.2 & 55.9 \\
\hline & & $C N N+C T D$ & & 60.0 & 81.5 & 69.1 \\
\hline & \multirow[t]{2}{*}{ Verga et al. [16] } & Transformer & \multirow[t]{2}{*}{ Doc_E } & 55.6 & 70.8 & 62.1 \\
\hline & & Transformer+ Extra data & & 64.0 & 69.2 & 66.2 \\
\hline \multirow[t]{6}{*}{ Tradional ML with KB } & Alam et al. [11] & $S V M+C T D+p p$ & Doc_E + Sen_M & 43.7 & 80.4 & 56.6 \\
\hline & Xu et al. [9] & SVM + CTD + SIDER+MEDI & Doc_E + Sen_M & 65.8 & 68.6 & 67.2 \\
\hline & Pons et al. [8] & SVM + Graph DB & Doc_E & 73.1 & 67.6 & 70.2 \\
\hline & \multirow[t]{2}{*}{ Peng et al. [7] } & SVM + CTD + Rules & \multirow[t]{2}{*}{ Doc_E } & 68.2 & 66.0 & 67.1 \\
\hline & & SVM + CTD + Rules +Extra data & & 71.1 & 72.6 & 71.8 \\
\hline & Lowe et al. [10] & rules+Ontology+WIKI+PP & Sen_M & 59.3 & 62.3 & 60.8 \\
\hline \multirow[t]{3}{*}{ NN without KB } & Gu et al. [13] & $\mathrm{CNN}+\mathrm{ME}+\mathrm{pp}$ & Doc_M + Sen_M & 55.7 & 68.1 & 61.3 \\
\hline & Zhou et al. [12] & LSTM+SVM + pp & Sen_M & 55.6 & 68.4 & 61.3 \\
\hline & Gu et al. [18] & ME & Doc_M + Sen_M & 62.0 & 55.1 & 58.3 \\
\hline
\end{tabular}

The 4-th column denotes the text level and the concept level when candidate instances are constructed. "Doc" denotes the document level, "Sen" denotes the sentence level, "_E" denotes entity-based candidate pairs and "_M" denotes mention-based candidate pairs. In addition, all results listed in this table come from the corresponding improved systems after the CDR challenge. The highest F-scores in each group of methods are highlighted in bold

integrated the semantic representation of an article into knowledge from CTD. Thus, the importance of different knowledge with respect to a special article is discerned. Moreover, their mention level system has to define heuristic rules to determine the final relation type of a candidate pair because the CDR corpus only provides the annotations at the entity level. In contrast, we not only designed the neural network architecture at the document level but also considered the contiguity and temporality among associated sentences as well as the theme of an article.

Table 8 lists recognizing results of two types of CID relations including the intra- and inter-sentential CIDs before and after knowledge is introduced into the proposed model. It has been observed from Table 8 that, in addition to the promotion of the precision and the

Table 8 The recognizing performance of the inter-sentential and intra-sentential CIDs before and after knowledge is introduced into the proposed model

\begin{tabular}{llllllll}
\hline Knowledge & CIDs & $\mathrm{P}(\%)$ & $\mathrm{R}(\%)$ & $\mathrm{F}(\%)$ & $\mathrm{TP}$ & $\mathrm{FP}$ & $\mathrm{POS}$ \\
\hline Without KB & inter-sentential & 45.0 & 42.9 & 43.9 & 130 & 159 & 303 \\
& intra-sentential & 59.5 & 77.9 & 67.4 & 594 & 405 & 763 \\
\multirow{2}{*}{ With KB } & inter-sentential & 52.8 & 60.1 & 56.2 & 182 & 163 & 303 \\
& intra-sentential & 68.8 & 83.4 & 75.4 & 636 & 289 & 763
\end{tabular}

The experiments were performed when the new development set is the subset with the index 0 (similarly hereinafter). TP, FP and POS denotes the number of predicted true positive instances, predicted false positive instances and true positive instances of the test dataset, respectively recall, F-scores of inter- and intra-sentential CID relations increase by 12.3 and $8.0 \%$, respectively, after knowledge is added into the proposed model. Hence, it might be inferred that the introduction of knowledge will help to further improve overall performances of recognizing complicated inter-sentential CID relations.

\section{(2) Comparison with tradition ML-based systems}

As shown in Table 7, NN-based systems obtain competitive performances compared with traditional-MLbased systems, most of which performed the recognition of CID relations by SVM classifier. Similar to Li et al. [17], these SVM-based systems didn't distinguish the importance of different relations from CTD on the candidate pair of a special article. In addition to directly and indirectly utilized knowledge features, they explored a great deal of features (approximately 20 types) including entity features, various context features and statistic features. Therefore, it can be observed from Table 7 that SVM-based systems generally achieve relatively high precisions due to elaborate feature selection. On the contrary, NN-based systems exploited fewer features besides the word embedding. For example, our model only used the PoS embedding, while $\mathrm{Li}$ et al. only employed the position embedding. As a result, NN-based systems generally obtained relatively high recalls. However, Table 9 indicates that the proposed model has the potential for growth of the precision and F-score with the increasing number of training samples. 
Table 9 The recognizing performance on the test dataset of the CDR corpus when different training sets were applied without postprocessing

\begin{tabular}{lllll}
\hline Training set & Knowledge & $\mathrm{P}(\%)$ & $\mathrm{R}(\%)$ & $\mathrm{F}(\%)$ \\
\hline Only original training set & Without KB & 45.2 & 68.1 & 54.3 \\
& With KB & 55.8 & 81.4 & 66.1 \\
\multirow{2}{*}{ Train +development set } & Without KB & 54.3 & 65.9 & 59.5 \\
& With KB & 61.5 & 78.7 & 69.0 \\
\hline
\end{tabular}

As for the running time, the proposed system took the server about 33 seconds to finish relation classification of CID on the CDR test set when it ran on the server equipped with 3 G CPU, 125G memory and 12GB TITAN Xp GPU. Undoubtedly, the SVM-based systems run more quickly than the NN-based systems in the context of the same hardware configurations. However, with the development of hardware technologies such as processor and memory technologies, the time performance will be no longer a main problem for the classification task.

On the whole, each of traditional ML-based and NN-based systems has its advantages and disadvantages. The traditional ML-based systems not only don't require too much training samples but also have the straightforward characteristic in the usage and the interpretability of features as well as less computational time, while the NN-based systems are able to partly automatically learn the high level representations of texts to reduce manual interventions if there are the moderate number of training samples.

\section{Conclusion}

In this work, we proposed an effective document level neural network model integrated domain knowledge for classifying complicated relationships between chemicals and diseases from biomedical articles. Depending on the learned semantic meaning of an article, the proposed system employed attention mechanism on domain knowledge to avoid learning representations of texts and knowledge in isolation to some extent. Experimental analyses indicate that the introduced knowledge attention has the ability to distinguish the effect of different knowledge on a special candidate pair and improves performances of the proposed system. Moreover, the proposed model constructed at the document level has more advantages over sentence level or mention level models for the recognition of inter-sentential CID relations. In spite of only three types of embedding vectors, experimental results on the CDR corpus show that the proposed system achieves a good overall performance compared with other state-of-the-art systems. Furthermore, the proposed model is flexibly scalable by replacing its document level sub-network with the other high-performance sub-network modules learning the document level semantic representation of an article. Essentially, the proposed model is easy to generalize to the analogous applications integrating domain knowledge.

\section{Endnotes \\ ${ }^{1}$ http://ctdbase.org/ \\ ${ }^{2}$ http://ctdbase.org/downloads/;jsessionid=9A \\ 140902CFAF161528E3DB29E4A70DCA\#cd \\ ${ }^{3}$ https://github.com/thunlp/Fast-TransX \\ ${ }^{4} \mathrm{ftp}$ ://ftp.ncbi.nlm.nih.gov/pub/lu/PubTator/ \\ ${ }^{5}$ https://code.google.com/p/word2vec/}

\section{Abbreviations}

BLSTM: Bidirectional long short term memory; CDRs: Chemical-disease relations; CID: Chemical-induced diseases; CNN: Convolution neural network; CTD: Comparative toxicogenomics database; DL: Deep learning; F: F-score; KB: Knowledge bases; LSTM: Long short term memory; MEDI: MEDication indication resource; MeSH: Medical subject headings; ML: Machine learning; NLP: Natural language processing; NN: Neural network; P: precision; PoS: Part of speech; R: Recall; RMSprop: Resilient Mean Square Propagation; RNN: Recurrent neural network; SIDER: Side effect resource; SVM: Support vector machine

\section{Acknowledgements}

We would like to thank the editors and all anonymous reviewers for valuable suggestions and constructive comments.

\section{Funding}

This work was supported by the Natural Science Foundation of China (No.61572102, No.61572098 and No.61502071), the Major State Research Development Program of China (No2016YFC0901900), the Natural Science Foundation of Liaoning provincial, China (No. 20180551078) and the Fundamental Research Funds for the Central Universities (No.DUT16RC(4)63). The funding bodies did not play any role in the design of the study, data collection and analysis, or preparation of the manuscript.

Availability of data and materials

The experimental data are available at https://github.com/vivianzw/CDR_KB

\section{Authors' contributions}

WZ carried out the overall algorithm design and experiments. HL participated in the algorithm design. XL and BX participated in experiments. All authors participated in manuscript preparation. All authors read and approved the final manuscript.

Ethics approval and consent to participate

Not applicable.

Consent for publication

Not applicable.

Competing interests

The authors declare that they have no competing interests.

\section{Publisher's Note}

Springer Nature remains neutral with regard to jurisdictional claims in published maps and institutional affiliations.

\section{Author details}

${ }^{1}$ College of Computer Science and Technology, Dalian University of Technology, Dalian, China. ${ }^{2}$ College of Software, Dalian JiaoTong University, Dalian, China. 
Received: 4 March 2018 Accepted: 14 August 2018

Published online: 17 September 2018

\section{References}

1. Islamaj Dogan R, Murray GC, Névéol A, Lu Z. Understanding PubMed ${ }^{\circledR}$ user search behavior through log analysis. Database. 2009;2009:bap018.

2. Névéol A, Doğan Rl, Lu Z. Semi-automatic semantic annotation of PubMed queries: a study on quality, efficiency, satisfaction. J Biomed Inform. 2011:44(2):310-8.

3. Davis AP, Grondin CJ, Lennon-Hopkins K, Saraceni-Richards C, Sciaky D, King BL, Wiegers TC, Mattingly CJ. The comparative Toxicogenomics Database's 10th year anniversary: update 2015. Nucleic Acids Res. 2014;43(D1):D914-20.

4. Wei C-H, Peng Y, Leaman R, Davis AP, Mattingly CJ, Li J, Wiegers TC, Lu Z. Assessing the state of the art in biomedical relation extraction: overview of the BioCreative V chemical-disease relation (CDR) task. Database. 2016;2016: baw032.

5. Davis AP, Wiegers TC, Roberts PM, King BL, Lay JM, Lennon-Hopkins K, Sciaky D, Johnson R, Keating $H$, Greene N. A CTD-Pfizer collaboration: manual curation of 88000 scientific articles text mined for drug-disease and drug-phenotype interactions. Database. 2013;2013:bat080.

6. Li J, Sun Y, Johnson RJ, Sciaky D, Wei C-H, Leaman R, Davis AP, Mattingly CJ, Wiegers TC, Lu Z. BioCreative V CDR task corpus: a resource for chemical disease relation extraction. Database. 2016;2016:baw068.

7. Peng $Y$, Wei $\mathrm{C}-\mathrm{H}$, Lu Z I Improving chemical disease relation extraction with rich features and weakly labeled data. J Cheminform. 2016;8(1):53.

8. Pons E, Becker BF, Akhondi SA, Afzal Z, Van Mulligen EM, Kors JA. Extraction of chemical-induced diseases using prior knowledge and textual information. Database. 2016;2016:baw046.

9. $\mathrm{Xu} \mathrm{J,} \mathrm{Wu} \mathrm{Y,} \mathrm{Zhang} \mathrm{Y,} \mathrm{Wang} \mathrm{J,} \mathrm{Lee} \mathrm{H-J,} \mathrm{Xu} \mathrm{H.} \mathrm{CD-REST:} \mathrm{a} \mathrm{system} \mathrm{for}$ extracting chemical-induced disease relation in literature. Database. 2016:2016:baw036.

10. Lowe DM, O'Boyle NM, Sayle RA. Efficient chemical-disease identification and relationship extraction using Wikipedia to improve recall. Database. 2016;2016:baw039

11. Alam F, Corazza A, Lavelli A, Zanoli R. A knowledge-poor approach to chemical-disease relation extraction. Database. 2016;2016.

12. Zhou H, Deng H, Chen L, Yang Y, Jia C, Huang D. Exploiting syntactic and semantics information for chemical-disease relation extraction. Database. 2016:2016:baw048

13. Gu J, Sun F, Qian L, Zhou G. Chemical-induced disease relation extraction via convolutional neural network. Database. 2017;2017(1). https://doi.org/10. 1093/database/bax024

14. Jiang Z, Jin L, Li L, Qin M, Qu C, Zheng J, Huang D. A CRD-WEL system for chemical-disease relations extraction. In: The fifth BioCreative challenge evaluation workshop; 2015. p. 317-26.

15. Li Z, Yang Z, Lin H, Wang J, Gui Y, Zhang Y, Wang L. CIDExtractor: A chemical-induced disease relation extraction system for biomedical literature. In: Bioinformatics and Biomedicine (BIBM), 2016 IEEE International Conference on IEEE. 2016:994-1001

16. Verga P, Strubell E, Shai O, Mccallum A. Attending to all mention pairs for ful abstract biological relation extraction. arXiv preprint arXiv: 171008312. 2017.

17. Li H, Chen Q, Tang B, Wang X. Chemical-induced disease extraction via convolutional neural networks with attention. In: IEEE International Conference on Bioinformatics and Biomedicine; 2017. p. 1276-9.

18. Gu J, Qian L, Zhou G. Chemical-induced disease relation extraction with various linguistic features. Database. 2016;2016:baw042.

19. Fillmore CJ. Frame semantics and the nature of language. Ann N Y Acad Sci. 1976:280(1):20-32

20. Minsky ML. Society of mind. Columbia: Simon \& Schuster; 1985

21. Ahn S, Choi H, Pärnamaa T, Bengio Y. A neural knowledge language model. arXiv preprint arXiv:1608.00318. 2016.

22. Yang B, Mitchell T. Leveraging knowledge bases in LSTMs for improving machine reading. In: Meeting of the Association for Computational Linguistics; 2017. p. 1436-46.

23. Toutanova K, Chen D, Pantel P, Poon H, Choudhury P, Gamon M. Representing Text for Joint Embedding of Text and Knowledge Bases. Proceedings of the 2015 Conference on Empirical Methods in Natural Language Processing: 2015

24. Hao Y, Zhang Y, Liu K, He S, Liu Z, Wu H, Zhao J. An end-to-end model for question answering over Knowledge Base with cross-attention combining global knowledge. In: Meeting of the Association for Computational Linguistics; 2017. p. 221-31.

25. Das R, Zaheer M, Reddy S, Mccallum A. Question answering on knowledge bases and text using Universal Schema and Memory Networks. arXiv preprint arXiv: 1704.08384. 2017:358-365.

26. Luong M-T, Pham H, Manning CD. Effective approaches to attention-based neural machine translation. arXiv preprint arXiv: 1508.04025. 2015.

27. Bahdanau D, Cho K, Bengio Y. Neural machine translation by jointly learning to align and translate. arXiv preprint arXiv: 1409.0473. 2014.

28. Wei Z, Lin H, Ling L, Zhao Z, Li Z, Zhang Y, Yang Z, Jian W. An attentionbased effective neural model for drug-drug interactions extraction. BMC Bioinform. 2017; 18(1):445

29. Wang L, Cao Z, de Melo G, Liu Z. Relation classification via multi-level attention cnns. In: Proceedings of the 54th Annual Meeting of the Association for Computational Linguistics Association for Computational Linguistics. 2016.

30. Zhang Y, Zheng W, Lin H, Wang J, Yang Z, Dumontier M. Drug-drug interaction extraction via hierarchical RNNs on sequence and shortest dependency paths. Bioinformatics. 2017;34(5):828-35.

31. Manning CD, Surdeanu M, Bauer J, Finkel JR, Bethard S, McClosky D. The stanford corenlp natural language processing toolkit. In: ACL (System Demonstrations); 2014. p. 55-60.

32. Bengio $Y$, Ducharme $R$, Vincent $P$, Jauvin $C$. A neural probabilistic language model. J Mach Learn Res. 2003:3(Feb):1137-55.

33. Elman JL. Finding structure in time. Cogn Sci. 1990;14(2):179-211.

34. Hochreiter S, Schmidhuber J. Long short-term memory. Neural Comput. 1997;9(8):1735-80

35. Collobert R, Weston J, Bottou L, Karlen M, Kavukcuoglu K, Kuksa P. Natural language processing (almost) from scratch. J Mach Learn Res. 2011;12(Aug): 2493-537.

36. Tieleman T, Hinton G. Lecture 6.5-rmsprop: Divide the gradient by a running average of its recent magnitude. COURSERA: Neural Networks for Machine Learning. 2012:4(2):26-31.

37. Zheng W, Lin H, Li Z, et al. An effective neural model extracting document level chemical-induced disease relations from biomedical literature. J Biomed Inform. 2018;83:1-9.

38. Bordes A, Usunier N, Weston J, Yakhnenko O. Translating embeddings for modeling multi-relational data. In: International Conference on Neural Information Processing Systems; 2013. p. 2787-95.

39. Wei C-H, Kao H-Y, Lu Z. PubTator: a web-based text mining tool for assisting biocuration. Nucleic Acids Res. 2013:41(W1):W518-22

40. Mikolov T, Sutskever I, Chen K, Corrado G, Dean J. Distributed representations of words and phrases and their compositionality. Comp Sci. 2013:3111-19.

41. Zeng D, Liu K, Lai S, Zhou G, Zhao J. Relation classification via convolutional deep neural network. In: Proceedings of COLING: 2014. p. 2335-2344.

42. Hinton GE, Srivastava N, Krizhevsky A, Sutskever I, Salakhutdinov RR. Improving neural networks by preventing co-adaptation of feature detectors. arXiv preprint arXiv: 1207.0580. 2012.

Ready to submit your research? Choose BMC and benefit from:

- fast, convenient online submission

- thorough peer review by experienced researchers in your field

- rapid publication on acceptance

- support for research data, including large and complex data types

- gold Open Access which fosters wider collaboration and increased citations

- maximum visibility for your research: over $100 \mathrm{M}$ website views per year

At BMC, research is always in progress.

Learn more biomedcentral.com/submissions 